\title{
On the spatial extent of attention in object-based visual selection
}

\author{
NILLI LAVIE \\ University College, London, England \\ and \\ JON DRIVER \\ Birkbeck College, London, England
}

\begin{abstract}
A new test was devised to avoid previous confounds in measures of object-based limits on divided visual attention. The distinction between objects was manipulated across a wide spatial extent. Target elements appeared on the same object only when far apart, and appeared close only when on different objects, so that object effects could not be reduced to spatial effects, nor vice versa. Subjects judged whether two odd elements within a display of two dashed lines were the same or different. They performed better when the target elements were far apart on a common line rather than on two distinct lines, even though the latter arrangement was more likely. Thus, nonstrategic object-based limits on divided attention can arise even across large distances. However, when subjects were precued to expect targets in a narrow region of the display, the object effect was eliminated, implying that object-based selection may only operate within spatially attended regions.
\end{abstract}

A recent controversy in attention research has been whether visual selection operates in a primarily spacebased or object-based manner (see, e.g., Duncan, 1984; Kanwisher \& Driver, 1992; Tsal \& Lavie, 1993). Numerous results show that spatial factors can influence normal visual attention. These include the well-known effects of spatial precuing (see, e.g., Posner, 1980), plus spatial constraints on distractor interference (Eriksen \& Hoffman, 1972), on visual feature miscombinations (Cohen \& Ivry, 1989), and on the ability to divide attention (Hoffman \& Nelson, 1981; Hoffman, Nelson, \& Houck, 1983). Treisman's influential feature-integration theory (e.g., Treisman \& Gelade, 1980) argues that spatial attention plays a special role in feature integration. Tsal and Lavie (1988, 1993) have proposed that visual selection of a stimulus distinguished by any target attribute (say, by its unique color or shape) is always effected by selecting the location of that distinguishing attribute.

Against this background of evidence for spatial effects on visual attention, and hypotheses about a possibly unique role for space, several authors have challenged the view that selection can be characterized as a purely spatial process. Duncan (1984) and Treisman, Kahneman, and Burkell (1983) proposed that attention is directed to segmented perceptual objects, rather than to unsegmented regions of

This research was supported in part by the Medical Research Council Applied Psychology Unit at Cambridge and a British Council award to the first author; and by a project grant from the Biotechnology and Biological Science Research Council to the second author. Our thanks to John Duncan, Art Kramer, Shaun Vecera, and an anonymous referee for helpful comments. Correspondence should be addressed to N. Lavie, Department of Psychology, University College London, Gower Street, London WC1E 6BT, England (e-mail: n.lavie@ ucl.ac.uk). space. A wide range of paradigms has since provided some support for grouping or object-based influences on visual selection. For instance, target-distractor grouping by common color, motion, or connectedness (Baylis \& Driver, 1992; Driver \& Baylis, 1989; Kramer \& Jacobson, 1991) can influence the extent of distractor interference. Precuing effects can be influenced by object-based factors as well as spatial factors (Egly, Driver, \& Rafal, 1994; Gibson \& Egeth, 1994; Vecera, 1994). Finally, the distinction between separate objects can apparently influence divided visual attention (Duncan, 1984; Treisman et al., 1983), as we shall review extensively below.

Despite this abundance of evidence for both spatial and object-based effects on visual selection, the broader theoretical picture remains far from clear. Spatial proximity will usually correlate highly with object distinctions, since elements of the same object are typically close together. For this reason, it is possible to reinterpret many apparently spatial effects in object-based terms (see Duncan, 1984) and vice versa (see Tsal \& Lavie, 1993). Furthermore, although both spatial and object-based effects have been found, the boundary conditions for each, and the precise relations between them, still remain unclear.

We will illustrate these points below with reference to previous experiments on the ease of dividing visual attention. Our paper focuses on just the divided-attention paradigm, since it provided some of the original evidence for object-based views (Duncan, 1984; Treisman et al., 1983) and has since been frequently cited in their support (e.g., Baylis, 1994; Baylis \& Driver, 1993; Duncan, 1993a, 1993b; Vecera \& Farah, 1994). We will discuss possible objections to previous reports of object-based limits on divided visual attention. This in turn will lead to our presentation of a new test for object-based limitations, de- 
signed with the intention that any spatial limitations should also be observable.

\section{Previous Evidence on Dividing Visual Attention}

Spatial restrictions on visual dual-task performance. Hoffman and Nelson (1981) had subjects judge either just the shape of a target letter, or just the orientation of a $U$, or both at the same time. Performance operating characteristics (POCs) were derived by systematically varying priorities for each task under divided attention. The critical manipulation was the spatial separation between the two objects that attention had to be divided across. The $U$ and the target letter were either very close $\left(.17^{\circ}\right.$ apart $)$ or quite distant $\left(3.0^{\circ}-4.3^{\circ}\right.$ apart $)$. POC analyses revealed an extensive tradeoff in dual-task performance when the two judgments concerned distant objects, yet negligible differences between single- and dual-task performance when the objects were close. This suggests that attentional resources can only be shared across a narrow spatial extent. Hoffman et al. (1983) reported further evidence consistent with this proposal from a similar paradigm.

Object-based restrictions on visual dual-task performance. Treisman et al. (1983) and Duncan (1984) originally reported that object factors also influence divided attention. Treisman et al. presented a rectangular frame whose outline was $1^{\circ}$ of visual angle from a word. Either the rectangle surrounded the word, or the two shapes were separated, with one above and one below fixation. Subjects were required to read the word and to judge the position (left vs. right) of a gap in the rectangular frame. The gap itself was as close to the word in the separated format as when the frame surrounded the word, although other parts of the frame were farther away. Dual-task performance was less efficient with the separated format.

Treisman et al. (1983) argued that the frame and word formed a single integral object in the surrounded format, but two distinct objects in the separated format, and they therefore attributed their results to an object-based limit on divided attention. However, it might be objected that the displays always comprised two distinct objects (i.e., the frame plus the word), with just their relative location varying (as in the Hoffman \& Nelson, 1981, or Hoffman et al., 1983, studies discussed above). Treisman et al.'s results would still show that performance depended on the location of the entire frame, not just the gap itself. However, it may be possible to explain this in purely spatial terms. The onset of the frame should provide an uninformative spatial cue (as in the covert orienting paradigm of Posner, 1980) that should impair reading in just the separated condition by drawing covert attention away from the word.

Duncan (1984) also argued for a difficulty in dividing visual attention between objects. He presented a 2-D outline box with a line struck through it, for brief exposures followed by a mask. Subjects had to judge one or two of the following attributes: line texture, line orientation, box height, or the locus of a gap in the box. Two attributes of the box, or two of the line, could be judged with minimal impairment compared with judging just one. There was more dual-task decrement for judging one attribute from the line and one from the box.

Duncan (1984) argued that these results must reflect a difficulty in dividing attention between objects, not locations, since the two objects were superimposed in roughly the same area, and the two box attributes were designed to be no closer to each other than to the line attributes. On the other hand, the two line attributes were always closest to each other, being literally defined across the same spatial locations, and Duncan $(1984$, p. 508$)$ did find that the easiest dual-task concerned the line. This hints at a possible role for spatial restrictions on divided attention, even in these experiments.

A more critical point was made by Watt (1988) and Baylis and Driver (1993). The two attributes of the line (texture and fine orientation) are primarily available at high spatial frequencies, whereas the two attributes of the box (height and gap) are available at low spatial frequencies. Thus, Duncan's (1984) results may reflect a difficulty in attending to different spatial frequencies, rather than to different objects or locations. Duncan (1993a, 1993b) has since conducted further studies with different stimuli and has again found a cost for dividing attention between objects versus within an object. However, the two objects were always in two different locations for these experiments, so the results are just as consistent with spacebased limits on divided attention (Hoffman \& Nelson, 1981; Hoffman et al., 1983) as with any object-based limit.

Baylis and Driver (1993) devised a way to compare oneobject and two-object judgments for identical displays, thus avoiding potential criticisms in terms of the locations or spatial frequencies of the critical stimuli. They used an ambiguous figure-ground display, analogous to Rubin's (1921) faces-vase engraving. Subjects' perceptual set was manipulated so that they either assigned two critical edges to a single common object (as with Rubin's vase) or assigned the very same edges to two different objects (as with Rubin's faces). Comparing the two edges was more difficult under the two-object interpretation (see also Baylis, 1994; Baylis \& Driver, 1995; Gibson, 1994).

These results apparently reveal a difficulty in judging two objects that cannot be attributed to differing spatial frequencies or locations. However, Baylis and Driver's (1993) results may be specific to the influence of figureground segmentation on edge assignment, rather than reflecting any general purpose limit on attention across objects. Indeed, Baylis and Driver (1993) suggest just this, arguing that comparisons of edges within a single figure are based on its shape, whereas comparisons of edges from two different figures cannot be based on such shape information alone and are thus less efficient.

Possible influences of space on object-based visual selection. Most reports of object-based limits to divided visual attention have stemmed from situations where the spatial separation between judged attributes was held constant and at a minimal level. For instance, in Duncan's (1984) study, the overlapping objects together subtended 
less than $0.5^{\circ}$. Such spatial compactness may have been dictated by the aim of demonstrating object-based effects that were not confounded with spatial separation. However, it leaves open the possibility that such object-based effects may only arise within narrow, spatially attended regions. Space-based theories of attention (e.g., Eriksen \& Hoffman, 1972, 1973) have long argued that visual attention can only be restricted to a finite extent (with about $1^{\circ}$ often cited as the limit, though see LaBerge, Brown, Carter, Bash, \& Hartley, 1991). If there is such a limit on the spatial restriction of attention, then Duncan's (1984) subjects may have been quite unable to select the relevant attributes of the box and line stimuli by purely spatial means. ${ }^{1}$

One can therefore argue that object-based factors may only become important within a hypothetical "spotlight" or "zoom-lens" of spatial attention, when no further spatial restriction is possible. Indeed, Mack, Tang, Tuma, Kahn, and Rock (1992) have recently argued that the distinction between separate objects is made only within the attended region, with no segregation of distinct objects taking place outside it. If so, spatial selection might be the rule, with object-based selection taking place only within narrow attended regions when no further spatial restriction is possible.

Vecera and Farah (1994) recently examined how objectbased effects on divided visual attention might depend on the spatial extent of the display. They repeated Duncan's (1984) study while manipulating the spatial separation of the line and box. These two objects now appeared either overlapping at fixation (as in Duncan's original study) or separated by $3.8^{\circ}$, with one on the left and one on the right of fixation. The results showed no interaction between the cost of dividing attention across objects and the spatial separation between them (although see Kramer, Weber, \& Watson, in press). This apparently suggests that spatial extent is irrelevant to object-based restrictions on attention, contrary to the possibility raised above.

While Vecera and Farah reported surprise at the null effect of separation, they suggested that it arose because the various judgments were all based on object-centered shape representations (Marr \& Nishihara, 1978), which do not code the position of the shapes. Indeed, they argued that truly "object-centered" selection should by definition be spatially invariant, and that it should apply whenever the judged attributes concern abstract shape properties (see also Baylis \& Driver, 1993). In further experiments, they used a task of dot detection, which should not require object-centered shape representations. They found that cuing the outline object (box vs. line) on which a dot subsequently appeared was more effective when the objects were separated in space. Evidently, attention did not operate in a spatially invariant manner for the dot detection task.

Vecera and Farah (1994) make clear logical distinctions between different senses of "object-based" attention, in introducing their own proposal of spatially invariant object-centered selection for shape attributes. However, a sceptic might question whether their results conclusively establish the latter form of selection. The absence of any separation effect with the Duncan (1984) box-and-line tasks is paradoxical for several reasons. Recall the study of Hoffman and Nelson (1981), which involved identifying a letter's shape while judging the orientation of a U. Those tasks seem just as shape-based as the Duncan tasks of judging line orientation, line texture, box height, or the location of the box's gap; yet substantial effects of spatial separation were found in the Hoffman and Nelson study, whereas there were none in the Vecera and Farah study, in which Duncan's tasks were used.

One potentially important procedural difference from Hoffman and Nelson's (1981) study is that Vecera and Farah (1994) did not control for eccentricity when varying spatial separation (see Kramer et al., in press) or for any effects of superimposition per se. Perhaps their comparison of foveal superimposed objects with peripheral separated objects is misleading. Superimposing the objects may produce lateral masking between them, or introduce some particular difficulty in attentional selection. Any such problems with superimposition would be eliminated when the objects were separated. The elimination of these difficulties might therefore mask any cost from having to divide attention across distinct and more eccentric locations in the separated condition.

Finally, the use of Duncan's (1984) box-and-line tasks means that all the two-object costs in the Vecera and Farah (1994) experiments could be attributed to the spatial frequency artifacts discussed earlier (Baylis \& Driver, 1993; Watt, 1988). If the difficulty in attending two objects was indeed due to a difference in their critical spatial frequencies, it might be less surprising that this cost was roughly maintained when the objects appeared farther apart.

Despite these potential criticisms, the Vecera and Farah (1994) study does raise the important question of whether object-based influences on divided attention vary with spatial separation. As noted earlier, most previous studies of object-based attention either have confounded object distinctions with spatial separation, or have scarcely manipulated separation, with the result that all the stimuli may have fallen within a spatially attended region.

\section{A New Test for Object-Based Limitations on Divided Attention}

In the light of the uncertainties discussed for previous evidence, we sought a new task that would allow further examination of any object-based limits on divided visual attention, while avoiding the potential pitfalls raised. Our first criterion was that the division between objects should be manipulated across a very wide spatial extent. This allows us to test whether object factors only dominate within narrow displays, and to measure both objectbased and space-based influences at the same time.

Our second criterion was that the elements to be judged within objects and between objects should be equivalent, to rule out stimulus artifacts such as the possible spatialfrequency problems with Duncan's (1984) box-and-line 
stimuli. Third, we sought a task that would not, by its intrinsic nature, force judgments to be dependent on objectbased distinctions or to be independent of them. To avoid intrinsic independence from objects, we considered it critical that the judged attributes should unequivocally appear as constituent elements of the objects in question (unlike, say, the dots in Vecera and Farah's cuing study [1994, Experiment 3], which were abruptly superimposed on preexisting outline objects, and thus were probably coded as distinct objects in their own right). To prevent any intrinsic dependence on objects, we avoided using judgments that would allow a relative comparison within objects but would require an absolute judgment across objects. As discussed by Baylis and Driver (1993), judgments of shape or of the position of shape elements may yield an inherent within-object advantage for these reasons.

Our fourth criterion was that the eccentricity, and thus acuity, for critical elements should be comparable for all conditions (cf. Vecera \& Farah, 1994). Finally, the instructions and task requirements should not specify attention to objects or locations in advance. Many previous experiments may, in effect, have "cued" the form of selection that took place. For example, instructing subjects to judge the orientation and texture of the line but not the box (Duncan, 1984) while presenting these two objects in the same location may effectively force subjects to attend a particular object rather than a particular location. Equally, informing the subject that a subsequent target is likely in a particular position on an otherwise empty screen (e.g., Posner, 1980) may effectively specify attention to location rather than to objects in advance. Unlike such previous studies, our intention was to manipulate both spatial separation and object factors substantially at the same time, while requiring subjects to make a judgment that did not make one factor intrinsically more relevant than the other.

\section{EXPERIMENT 1}

The stimuli for each trial comprised two long dashed lines which were briefly presented together. The task was to compare two odd elements within each such display (each odd element comprised a dot or a gap that replaced a standard dash), for a speeded judgment of whether these target elements were the same or different. The two target elements could be far apart, either in the same line (object condition) or in different lines ( far condition); or they could be very close together but in different lines (near condition; see Figure 1). By comparing the efficiency of judgments in the near and far conditions, we could examine any spatial constraints on attention. By comparing judgments for the object and far conditions, we could examine any object-based restrictions on attention.

Note that this novel task satisfies the five criteria discussed earlier. First, since the displays subtended $13^{\circ}$, and since the target elements could be from $1^{\circ}$ to $8^{\circ}$ apart, depending on condition, the division between objects was manipulated across a wide spatial extent, while the spa-
NEAR condition, 'different' response (dot plus gap)

a)

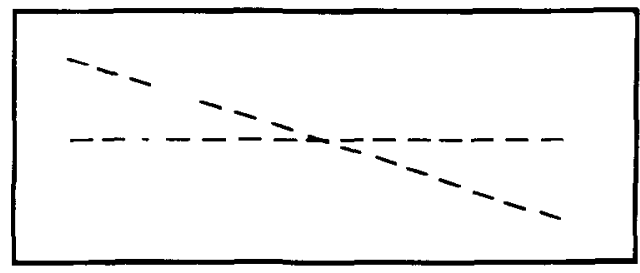

OBJECT condition, 'same' response (two gaps)

b)

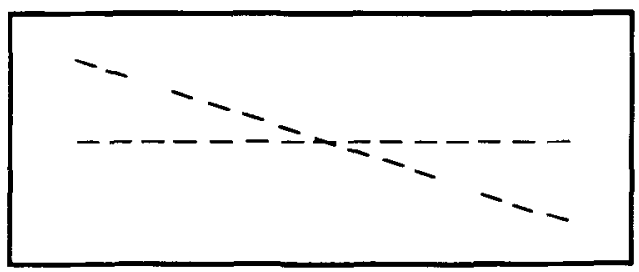

FAR condition, 'same' response (two dots)

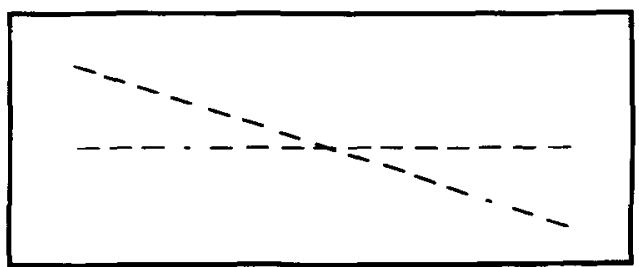

Figure 1. Example stimuli from Experiment 1. (a) An example of the near condition, with the two target elements close together in distinct objects and appearing toward the left. In this instance, they are different, but they were equally likely to be the same. (b) An example of the object condition, with target elements far apart on a common line. (c) An example of the far condition, with target elements far apart on distinct lines. Note that in each display the two lines were presented in different colors.

tial separation of the judged attributes was also manipulated considerably. Second, the elements to be judged (dots or gaps) were equivalent for our within-object and between-object conditions, thus avoiding possible stimulus confounds involving spatial frequency, and so forth.

Third, the required comparison of target elements seems to avoid some of the potential problems with previous tasks that might have led to intrinsic independence or dependence on object factors. Intrinsic independence may result if the target elements appear distinct from the one or two objects of which they are intended to be parts (as perhaps with Vecera \& Farah's, 1994, dot detection task). The present target elements certainly appear to be parts of the critical objects; that is, they belong to one of the two dashed lines as constituent elements. According to Baylis and Driver (1993), intrinsic dependence on object factors may result if the within-object judgment can be made in relative terms, whereas the between-object judg- 
ment must be made in absolute terms. This did not apply for the present task.

Fourth, the target elements appeared at equivalent eccentricities across all conditions in the present task, so we could manipulate their separation while avoiding acuity confounds (cf. Vecera \& Farah, 1994). Finally, the instructions and task requirements did not specify attention to objects or locations in advance. The target elements were distinguished just by their length (dots or gaps among the standard dashes that made up the two lines). Which objects the target elements would appear in, and at which locations, were equally unknown in advance.

\section{Method}

Subjects. The 10 subjects were paid volunteers who participated in exchange for $£ 4.00$. Their age ranged from 18 to 33 years, and all reported normal or corrected vision.

Apparatus and Stimuli. The stimuli were presented, and responses recorded, using an IBM-PC-compatible computer attached to a VGA color monitor. The experiments were created and run by Micro Experimental Laboratory (MEL; Schneider, 1988).

The stimuli for each trial were one red dashed line and one green dashed line which intersected at their midpoints at the center of the display, against a gray background. Both lines were straight, with one horizontal and the other tilted $18^{\circ}$ clockwise from the horizontal (see Figure 1). The horizontal and tilted lines were equally likely to be red and green, respectively, or vice versa, and this was unpredictable across trials. The horizontal line was $17.88 \mathrm{~cm}$ long, and the tilted line, $18.86 \mathrm{~cm}$ long. At the fixed viewing distance of $80 \mathrm{~cm}$, they subtended $12.6^{\circ}$ and $13^{\circ}$ of visual angle, respectively. Each line was composed of 15 elements that were evenly spaced. The standard dashes were $0.8 \mathrm{~cm}$ in length and two pixels in height. Adjacent standard dashes were separated by $0.42 \mathrm{~cm}$ for the horizontal line and $0.49 \mathrm{~cm}$ for the tilted line.

In every display, two of the standard dashes were replaced to provide two target elements. These comprised dots or gaps. A dot was $0.2 \mathrm{~cm}$ in diameter and was located as for the center of the dash that it replaced. A gap element was made simply by eliminating one of the standard dashes. Any dots or gaps were always located at the periphery of the display, as the third or fourth element from one end of a dashed line. Within each display, one target element was always the third from one end of a line, while the other was the fourth element from the end of a line. These slightly different positions within a line were used to avoid any unintended symmetries or potential subjective contours that might otherwise arise between the target elements in some conditions. Targets were equally likely to appear as the third or fourth element from the end for both the tilted line and the horizontal line.

The two target elements in each display were equally likely to be the same or different, and, when the same, to be both dots or both gaps. They were also equally likely to appear in the horizontal or in the tilted line. Finally, they were equally likely to appear at opposite ends of the same line (object condition); or a comparable distance apart at opposite sides of the screen, but now in distinct lines ( far condition); or in distinct lines but close together on the same side of the screen (near condition; see Figure 1).

The distance between target elements (measured between the center of two dots for convenience) was $1.3^{\circ}$ or $1.6^{\circ}$ in the near condition (depending on whether the target in the tilted line was the third or fourth element from one end of this line). The distance between targets was $7.8^{\circ}$ in the far condition and $8.2^{\circ}$ in the object condition. Thus, the separation of the judged elements was roughly comparable for the far and object conditions (with any spatial constraints on attention slightly favoring the far condition).

Combining all the possible variations in display type (horizontal line red or green; target elements same or different; whether each particular member in a pair of target elements was a dot or gap; whether each particular target was third or fourth from the end of the line containing it; and whether the relative spatial arrangement of the two targets corresponded to the near, far, or object condition) resulted in 48 possible displays.

Procedure. Subjects viewed the screen in a darkened room with their heads in a chinrest. Each trial began with a white fixation point appearing at the center of a gray screen for $1 \mathrm{sec}$. This was immediately followed by a display of two intersecting dashed lines, containing two odd target elements as described above, which appeared for $177 \mathrm{msec}$ (i.e., too briefly to permit saccades during its exposure). Subjects had to make a speeded judgment concerning whether the two target elements were the same (i.e., two dots or two gaps) or different (i.e., a dot and a gap). They responded with the right hand, using the numerical keypad on the right of the standard computer keyboard, pressing the " 0 " key with the thumb to indicate a same judgment, or the " 2 " key with the index finger to indicate a different judgment. Feedback was immediately given for errors by a 500 -msec computer tone.

The subjects were told that the two odd elements would appear at the periphery of the display, located in all possible pairwise combinations of the four ends of the lines. That is, the particular two target positions for each trial were uncertain, but it was known that targets could appear only toward the ends of the lines. The displays were presented in a random intermixed order in blocks of 64 trials, allowing the subjects to rest for a short while between blocks. There were 11 blocks in total, with the first discarded as practice. The 48 possible displays were equally likely. An intermission of 5 min was given after the first 6 blocks. The subjects initiated each block by pressing the space bar.

\section{Results}

Median reactions times (RTs) and percentage accuracy rates were computed for each subject for each condition of target arrangement (i.e., near, far, and object conditions) and for each required response (same vs. different ). The average of these RTs and accuracy rates across subjects is given in Table 1.

A two-way within-subjects analysis of variance (ANOVA) of the median RT data, with the factors of condition ( 3 levels) and response ( 2 levels) showed a main effect of response $[F(1,9)=21.1, p<.001]$, with slower different responses. There was also a main effect of condition $[F(2,8)=9.2, p<.01]$, and an interaction of condition with response $[F(2,8)=8.3, p<.01]$. This interaction can be explained by reference to the planned contrasts of the near condition and of the object condition with the far condition, which were of primary interest for the hypotheses under examination.

The planned comparison of RTs for object versus far conditions showed a significant effect $[F(1,9)=18.5$, $p<.01]$. Judgments were significantly faster for distant

Table 1

Mean Reaction Times (in Milliseconds) and Accuracy Rate (Percentage) Across Subjects $(n=10)$ as a Function of Condition and Response in Experiment 1

\begin{tabular}{|c|c|c|c|c|c|c|}
\hline \multirow[b]{3}{*}{ Response } & \multicolumn{6}{|c|}{ Condition } \\
\hline & \multicolumn{2}{|c|}{ Near } & \multicolumn{2}{|c|}{ Object } & \multicolumn{2}{|c|}{ Far } \\
\hline & RT & $\%$ & RT & $\%$ & RT & $\%$ \\
\hline Same & 708 & 86 & 630 & 95 & 665 & 94 \\
\hline Different & 748 & 91 & 717 & 94 & 737 & 92 \\
\hline
\end{tabular}


elements on a common line than for distant elements on distinct lines (see Table 1), even though their separation was roughly comparable (being slightly greater for the $o b$ ject condition). This outcome is consistent with previous claims of a difficulty in attending to attributes from two distinct objects (e.g., Duncan, 1984). The present object effect (i.e., the object vs. far comparison) did not interact with the required response $[F(1,9)=1.65$, n.s.].

By contrast, the planned near versus far comparison on RTs did show an interaction with response $[F(1,9)=$ $5.2, p<.05]$. There was no reliable difference between near and far conditions for different judgments $(F<1)$. However, the near condition was slower than the far for same responses $[F(1,9)=7.3, p<.02]$.

Comparable analyses were conducted on the accuracy data. A two-way omnibus ANOVA (condition $\times$ response) found a main effect of condition $[F(2,8)=5.6, p<.03]$, but no effect of response $(F<1)$ and no interaction $[F(2,8)=3.0$, n.s. $]$. The planned contrast of object and far conditions showed a trend for greater accuracy in the object condition $[F(1,9)=3.5, p<.10]$, which did not interact with response $(F<1)$. While not reaching significance, this trend for better performance in the object condition supports the pattern of results in the RT data, ruling out any speed-accuracy tradeoff as an explanation for the object effect.

The planned comparison of near and far conditions showed an interaction between response and condition $[F(1,9)=6.5, p<.03]$. As for the RT data, there was no difference between near and far conditions for different judgments $(F<1)$, but performance was worse in the near condition for same judgments $[F(1,9)=11.7, p<.01]$.

\section{Discussion}

In the present task, the location of the two target elements was unknown in advance, and it was likewise unknown whether they would appear in a common object or in distinct objects. Comparisons of these elements were faster, and tended to be more accurate, if the target elements belonged to a common object (i.e., a single line formed by the good continuation of aligned dashes) rather than to distinct objects (i.e., two different dashed lines).

This object effect is consistent with previous claims of a difficulty in attending to distinct objects (see, e.g., Duncan, 1984). Unlike previous reports of such a constraint, the present object effect cannot be attributed to different spatial frequencies, and so forth, for the withinobject versus between-object judgments, since the elements to be compared were equivalent across our object and far conditions.

Moreover, unlike in previous studies, the task requirements did not specify in advance that one target object should be attended regardless of its location. Instead, the present object effect arose spontaneously even though the distinction between objects was totally irrelevant to the prescribed task. The present findings also demonstrate that object-based constraints on attention can apply even to large displays. Previously, one could have argued that object-based influences on divided attention only predominate when displays are too narrow to permit any spatial restriction of attention within them. However, in the present study, the object effect was found when the target elements appeared on opposite sides of fixation, about $8^{\circ}$ apart.

Our use of wide displays meant that we were able to manipulate the spatial separation of target elements substantially when they belonged to distinct objects. Spacebased theories of attention (e.g., Eriksen \& Hoffman, 1972; Hoffman \& Nelson, 1981) would predict more efficient performance when the target elements were closer together. However, somewhat surprisingly, no advantage was found for the near condition over the far condition, even though the separation of the target elements was only $1.3^{\circ}-1.6^{\circ}$ in the former case, but $7.8^{\circ}$ in the latter case. In fact, the opposite pattern of a cost for the near condition was found when the target elements were of the same type (i.e., both dots or both gaps), whereas there was no effect of spatial separation when the targets were of different types. We will consider possible accounts for this interaction with response later. In our next experiment, we examined a possible reason why no overall near advantage was found, while also seeking to replicate the object effect.

\section{EXPERIMENT 2}

The near, far, and object conditions were equally likely in Experiment 1. This meant that the target elements were more often on opposite sides of the display (in the far and object conditions) than on the same side (in just the near condition, on only $1 / 3$ of the trials). This might have led to a strategy of selecting information from the opposite side of the display once a single target element had been found, which could have disadvantaged the near condition.

Note that the event probabilities in Experiment 1 were equally weighted against any strategy of attending to individual objects (i.e., to an entire dashed line), since the two target elements appeared in the same line on only $1 / 3$ of trials (the object condition), appearing in different lines for the remainder (the near and far conditions). Thus, although the event probabilities might have been weighted against finding a near advantage, they similarly did not favor an object advantage; yet the latter object-based effect was observed.

It might be, however, that location-based selection is particularly sensitive to event probabilities (see, e.g., Eriksen \& Yeh, 1985). Accordingly, in our next study we doubled the proportion of near trials, so that the two target elements in each display were now just as likely to be on the same side as on opposite sides. We thought that this might reveal an advantage for the near over the far condition. Note that the object condition now made up only $25 \%$ of all trials. That is, the two target elements were in different objects for $75 \%$ of the trials (as compared with $66 \%$ in the previous study). Thus, the probabilities were now weighted even more strongly against objectbased selection. Replicating the object-based effect under 
the present circumstances would therefore strongly imply that it is nonstrategic.

\section{Method}

Subjects. The 13 new subjects were volunteers who were paid as before and fell in the age range of 18-33 years. All reported normal or corrected vision.

Apparatus, Stimuli, and Procedure. The apparatus, stimuli, and procedure were identical to those of Experiment 1, except that each block of 64 trials now comprised 32 displays in the near condition, 16 in the far condition, and 16 in the object condition. Thus, the near condition now appeared twice as often as before.

\section{Results}

Data from one exceptionally slow subject were excluded (this subject had an average RT of $1,148 \mathrm{msec}, 2.3$ standard deviations from the rest of the group). The intersubject means of median RTs after this exclusion, together with the associated mean accuracy rates, are shown in Table 2 for the three conditions of interest, separated also by the required response.

A two-way ANOVA [condition (3) $\times$ response (2)] of the RT data showed a main effect of response $[F(1,11)=$ $27.7, p<.001]$, with slower different judgments than same judgments as in Experiment 1 . There was a main effect of condition $[F(2,10)=12.7, p<.002]$ and an interaction between condition and response $[F(2,10)=4.0$, $p<.05]$. This shows a pattern related to that found in Experiment 1 , as revealed by the planned contrasts on RTs for object versus far conditions, and for near versus far conditions.

Specifically, the object condition was significantly faster than the far condition $[F(1,11)=27.6, p<.001]$, just as in Experiment 1. This object-based effect did not interact with response $[F(1,11)=1.8$, n.s.]. By contrast, the comparison of near and far conditions showed a tendency to interact with response $[F(1,11)=3.5, p<.09]$, as in Experiment 1. The near condition was now faster than the far condition for different judgments $[F(1,11)=$ $5.3, p<.04]$, but not for same judgments $(F<1)$. Experiments 1 and 2 thus differ in that a near advantage was found in the present experiment (for different judgments) whereas a near disadvantage (for same judgments) had been found previously. However, in both cases, the interaction with response arose because the near condition showed less of a benefit for same over different judgments.

Comparable analyses were performed on the accuracy rates. A two-way omnibus ANOVA [condition $(3) \times$ re-

Table 2

Mean Reaction Times (in Milliseconds) and Accuracy Rate (Percentage) Across Subjects $(n=12)$ as a Function of Condition and Response in Experiment 2

\begin{tabular}{|c|c|c|c|c|c|c|}
\hline \multirow[b]{3}{*}{ Response } & \multicolumn{6}{|c|}{ Condition } \\
\hline & \multicolumn{2}{|c|}{ Near } & \multicolumn{2}{|c|}{ Object } & \multicolumn{2}{|c|}{ Far } \\
\hline & $\mathrm{RT}$ & $\%$ & $\overline{\mathrm{RT}}$ & $\%$ & RT & $\%$ \\
\hline Same & 658 & 86 & 625 & 92 & 661 & 90 \\
\hline Different & 706 & 90 & 714 & 88 & 733 & 85 \\
\hline
\end{tabular}

sponse (2)] found no effect of response $[F(1,11)=2.0$, n.s.] or of condition $[F(2,10)=2.2$, n.s.]. There was a significant interaction between condition and response $[F(2,10)=7.9, p<.01]$. As in the RT analysis, this interaction arose because the near versus far comparison varied as a function of response. Indeed, the planned near versus far comparison found an interaction between condition and response $[F(1,11)=15.0, p<.01]$. In accordance with the RT data, performance was better in the near condition than in the far condition for different judgments $[F(1,11)=6.6, p<.02]$. By contrast, there was a marginal tendency for poorer accuracy in the near condition for same judgments $[F(1,11)=4.4, p<.06]$. The planned contrast of accuracy for object versus far conditions did not reveal any significant effect of condition $[F(1,11)=2.8$, n.s. $]$, or any interaction with response $(F=1)$, although the trend was for better performance in the object condition for both responses, in agreement with the RT data (see Table 2).

\section{Discussion}

The most important finding in Experiment 2 was that the object-based effect from Experiment 1 was replicated. Judgments were faster and tended to be more accurate if the elements belonged to a common object rather than distinct objects, just as in Experiment 1 . The objectbased effect was found again, even though the target elements were now three times as likely to belong to distinct objects (in the near and the far conditions) as to a common object (in just the object condition). These event probabilities provide no strategic motivation for objectbased selection, but rather are weighted against it. The present findings therefore suggest that the tendency to select elements from one object together was nonstrategic in the present task.

While the object-based effect was constant across Experiments 1 and 2, the change in the event probabilities did affect our measure of spatial effects. In the present study, the near condition was made twice as likely, so that the two target elements within each display would just as often appear on the same side as on opposite sides of fixation across the experiment. The near condition was now superior to the far condition for different judgments, but there was no such pattern for same judgments; indeed there was a tendency for the near condition to be less accurate than the far when a same judgment was required. This aspect of the present near results is consistent with Experiment 1, in which the near condition was found to be disadvantaged relative to the far conditions for same judgments. It suggests that some factor produced a tendency to respond different for all near trials, which means that the present near advantage for different judgments could only be taken as suggestive evidence for spacebased selection.

We will return to discuss the possible effects of spatial separation later. For the moment, our next experiment focused on an alternative account of our clearest finding to date-namely, the object-based effect. 


\section{EXPERIMENT 3}

The two dashed lines that comprised each display in Experiments 1 and 2 were always presented in different colors (one red and one green). This was done in an effort to ensure that the two lines appeared as distinct objects, rather than, say, as a single $\mathrm{X}$ shape (it was for this same reason that one line was made horizontal and the other tilted). However, the use of two colors unfortunately allows an alternative explanation of the object-based effect.

Specifically, it might have been easier to compare target elements within a single line because they were in the same color, rather than because they were in the same object. Comparing target elements from different lines might have been harder just because they were in different colors. This potential criticism can clearly be made for the dot targets. One might try to argue that the gap targets were effectively always in the same "color" (i.e., they were always the gray shade of the blank screen that the lines were presented against). However, gaps are defined only by the elements that surround them, and these were the same color within one line but different colors between lines, so the objection may apply to gap targets as well.

Accordingly, in our next study we sought to replicate the object effect by using target elements that were always in the same color, regardless of whether or not they fell within the same line. The two dashed lines were still presented in different colors (their standard elements were now either pink or yellow), to ensure that they would be seen as distinct objects. However, the two odd target elements within each display were now always presented in white. The dot targets were just as before, except for being white. The gap targets were now replaced with a dash target--namely, a standard dash presented in white. The nontarget elements remained standard dashes as before, but were presented in pink or yellow (rather than the previous red or green) because pilot studies showed that these two new colors were equally distinguishable from white (whereas the white was more distinct from the previous red than from the previous green).

\section{Method}

Subjects. The 13 new subjects were volunteers, paid as before, and again falling in the age range of 18-33 years. All reported normal or corrected vision.

Apparatus, Stimuli, and Procedure. The apparatus, stimuli, and procedure followed those of Experiment 2 exactly, except that the nontarget dashes were now presented in pink for one line, and in yellow for the other, whereas the target elements were always white dots, or white dashes of the same standard length as that of the nontarget dashes. Subjects had to judge whether the two white elements in each display were the same (both dashes or both dots) or different (a dot and a dash). The horizontal and tilted lines were equally likely to be yellow and pink, respectively, or vice versa.

\section{Results}

The RTs and accuracy rates are shown for each condition in Table 3, averaged across subjects and separated
Table 3

\begin{tabular}{|c|c|c|c|c|c|c|}
\hline \multirow[b]{3}{*}{ Response } & \multicolumn{3}{|c|}{ Condition } & & & \\
\hline & \multicolumn{2}{|c|}{ Near } & \multicolumn{2}{|c|}{ Object } & \multicolumn{2}{|c|}{ Far } \\
\hline & RT & $\%$ & RT & $\%$ & RT & $\%$ \\
\hline ne & 630 & 91 & 614 & 95 & 625 & 92 \\
\hline Different & 657 & 92 & 641 & 93 & 662 & 93 \\
\hline
\end{tabular}

by the required response. A two-way omnibus ANOVA [condition (3) $\times$ response (2)] on the RT data showed a main effect of response $[F(1,12)=15.4, p<.01]$, with slower different judgments than same judgments as before. There was also a marginal effect of condition $[F(2,11)$ $=3.4, p<.07]$, but no interaction between condition and response $(F<1)$. Planned comparisons of the object and far conditions for RTs showed faster performance in the object condition, as in the previous experiments $[F(1,12)=$ $6.0, p<.03]$. The planned comparison of near versus $\mathrm{far}$ conditions showed no effects $(F<1)$.

The omnibus two-way ANOVA [condition $(3) \times$ response (2)] of the accuracy data did not show any significant results except for a trend toward an effect of condition $[F(2,11)=2.9, p<.10$; all other $F \mathrm{~s}<1]$.

\section{Discussion}

The important finding of the present experiment is that the object-based effect, as found in Experiments 1 and 2, was replicated once again, even though the target elements were now always in the same white color regardless of whether or not they fell in the same line. Thus, the object-based effect is not simply caused by a benefit for comparing elements in the same versus a different color. As before, the advantage for target elements within a common line was found even though the target elements were three times as likely to appear in distinct lines, again suggesting that the object-based effect is nonstrategic.

There were no reliable differences between the near and far conditions in the present experiment, even though the same event probabilities were used as in Experiment 2, where an advantage for the near condition had been observed in RTs for different judgments. Moreover, the present data did not show the interaction between near versus far condition and response that had characterized the effects of spatial separation in Experiments 1 and 2 (namely, the smaller benefit for same judgments over different judgments in the near condition as compared with the others).

One possible account would be that same judgments were problematic in the near condition for Experiments 1 and 2 because the target elements had different colors when in distinct objects for those studies. This difference in color might have been particularly striking when the target elements were close together (i.e., in the near condition), thus producing a tendency to respond different for the near condition even when the targets were actu- 
ally of the same type (see Dixon \& Just, 1978; Miller \& Bauer, 1981). This would impair same judgments relative to different judgments for the near condition in Experiments 1 and 2 . It would also fit the absence of any interaction with response for the near effects in the present experiment, because now the target elements always had the same white color.

We did not consider this suggestion worth pursuing any further, since the interaction of near effects with response in Experiments 1 and 2 was both unpredicted and irrelevant to the theoretical issues under examination. The important point from our first three experiments is that robust evidence for object-based constraints on dividing attention could be found in our new task, which was devised to avoid possible criticisms of previous objectbased findings. On the other hand, evidence for constraints from spatial proximity has been scant so far.

\section{EXPERIMENT 4}

The previous three experiments demonstrate that objectbased factors can influence divided visual attention even when the display covers a very wide extent. Our use of such large displays was motivated by the observation that previous reports of object-based attentional limits (e.g., Duncan, 1984) had typically resulted from the use of displays so small that spatial selection might have been impossible. Object-based selection in such studies might only have taken place within an attended region, when no further spatial restriction was possible.

By contrast, our displays should certainly have been large enough (at $13^{\circ}$ wide) to permit spatial attention to be restricted within them, given a range of previous evidence (e.g., Eriksen \& Hoffman, 1972). However, although our displays were physically large, subjects might have attempted to distribute their spatial attention right across them, giving their attentional "zoom-lens" (Eriksen \& St. James, 1986) a wide initial setting. This would certainly have been the sensible strategy, since the target events were equally likely to be at either extreme of the display, and the displays were too brief to allow focused serial search during exposure.

Thus, although Experiments 1-3 showed that objectbased selection could arise even in very large displays, it might be that this still operated only within spatially attended areas. In enlarging the size of the display as compared with that in previous studies, we might also have increased the size of the attended region in a corresponding fashion. This could explain the scarcity of effects from spatial proximity in our experiments thus far. If spatial attention were initially set to cover a wide area, one might not expect enhanced performance when the target elements were closer to each other (as in the near condition), since attention would not initially be focused on either element.

The object-based effect we repeatedly found suggests that attention tends to select elements from a common object. However, in the present experiments, such selec- tion could conceivably have taken place in a spatial manner, with an initially diffuse setting of spatial attention coming to focus in on the positions occupied by just one of the two objects (see the General Discussion for further elaboration of this possibility). Thus, spatial aspects of attention could have been involved in selection for the present task, perhaps operating on a grouped spatial array (see Kramer \& Jacobson, 1991; Vecera, 1994; Vecera \& Farah, 1994). This might apply without necessitating any benefit for near versus far elements on different objects.

Given the large extent of our displays, we were now in a position to manipulate the scale of spatial attention relative to the scale of the stimulus, to examine whether object-based selection would arise only within the spatial focus of attention. This was not possible in most previous studies of object-based limits to divided attention (e.g., Duncan, 1984), because the small size of the displays precluded it. In several prior studies using other attentional paradigms, such as distractor interference or cuing measures, spatial factors were varied orthogonally to object factors (e.g., Kramer \& Jacobson, 1991; Vecera, 1994). However, in these studies spatial distances were simply manipulated in the stimulus, rather than the spatial extent of attention for a given display being manipulated.

Hence our next experiment is the first to examine whether object-based attention is affected when subjects adopt a narrower spatial focus of attention for the same display. Specifically, in the final experiment we tested whether the object-based effect from the three previous experiments would still be found when subjects adopted a narrow focus of covert spatial attention. Subjects were now cued to concentrate on just one side of the display, rather than having a diffuse initial focus across the whole display as was encouraged by the event probabilities in Experiments 1-3.

The task was just as for Experiment 3-that is, comparing the two white elements in a display of two dashed lines whose nontarget elements were all pink or all yellow. As before, these target elements could be the same (two dots or two dashes) or different (a dot and a dash). The three possible arrangements of the targets (near, far, and object) were also as before. However, a precue now indicated the side where the target elements were both most likely to appear, thus allowing subjects to focus their covert attention narrowly within the display.

The two target elements both appeared on the cued side for $70 \%$ of all trials (valid-near condition). For $10 \%$ of the trials, they both appeared on the other side (i.e., on the uncued side but equally near to each other; the invalid-near condition). The remaining trials were split into $10 \%$ with the previous object arrangement (now invalid-object) and $10 \%$ with the previous far arrangement (invalid-far). One target element appeared on the cued side and one on the uncued side for both these conditions.

We expected better performance with the near arrangement on the cued rather than the uncued side. This would demonstrate that subjects had indeed focused 
their covert attention on the cued side. Our new question was whether the object-based effect found repeatedly in the previous three experiments (and now measured by the comparison of invalid-object and invalid-far performance) would be replicated under the new situation of narrowly focused covert spatial attention.

\section{Method}

Subjects. The 20 new subjects were again paid volunteers in the age range of 18-33 years, reporting normal or corrected vision.

Apparatus, Stimuli, and Procedure. The apparatus was as before. The stimuli and procedure were the same as in Experiment 3, except for the following changes. The dashed-line displays were now presented for only $130 \mathrm{msec}$, and they were preceded on each trial by a cue display that appeared for $70 \mathrm{msec}$. The cue display consisted of the endmost standard dash for both lines on either just the left or just the right of the display (i.e., two standard dashes in total, on just one side of the screen). These segments were also present during the subsequent dashed-line display. Their earlier onset caused an attention-capturing "flicker" on their side. Note that the interval from cue onset to display offset was too brief to permit any overt rather than covert shifts of attention.

The cue was valid on $70 \%$ of trials. That is, it was followed on $70 \%$ of trials by a dashed-line display with the white target elements having the near arrangement within distinct lines on the side of the cue (valid-near). The remaining trials were equally likely to have targets in the near arrangement on the uncued side (invalidnear); or in the far arrangement with the target elements in distinct lines and on opposite sides (invalid-far); or in the object arrangement with the target elements in a common line but on opposite sides (invalid-object).

Same and different responses were required equally often for each of these four conditions. Each block of 80 trials had 8 displays from each of the three invalid conditions, plus 56 displays from the valid-near condition. In all cases, the cued side was equally likely to be on the left or the right. Each subject underwent 1 I blocks of 80 trials, preceded by a short block of 12 example trials which the subjects simply watched. Only the last 800 trials were analyzed for each subject.

The subjects were instructed to pay attention to the side of the cue, since the two white target elements would appear on that side for most of the trials. However, they were also told that on a small proportion of trials at least one target, and possibly both targets, would appear on the uncued side; and they should try to respond as rapidly and accurately as possible for those trials as well. Otherwise their task was just as in the preceding three experimentsthat is, to judge whether the two odd elements in each display were the same or different.

\section{Results}

The RTs and accuracy rates (averaged across subjects) are shown for each of the four conditions in Table 4, sepa-

Table 4

Mean Reaction Times (in Milliseconds) and Accuracy Rate (Percentage) Across Subjects $(n=20)$ as a Function of Condition and Response in Experiment 4

\begin{tabular}{|c|c|c|c|c|c|c|c|c|}
\hline \multirow[b]{4}{*}{ Response } & \multicolumn{8}{|c|}{ Condition } \\
\hline & \multirow{2}{*}{\multicolumn{2}{|c|}{$\begin{array}{l}\text { Valid } \\
\text { Near }\end{array}$}} & \multicolumn{6}{|c|}{ Invalid } \\
\hline & & & \multicolumn{2}{|c|}{ Near } & \multicolumn{2}{|c|}{ Object } & \multicolumn{2}{|c|}{ Far } \\
\hline & RT & $\%$ & $\mathrm{RT}$ & $\%$ & $\mathrm{RT}$ & $\%$ & $\mathrm{RT}$ & $\%$ \\
\hline Same & 647 & 88 & 667 & 86 & 728 & 77 & 709 & $\overline{81}$ \\
\hline Different & 668 & 88 & 700 & 86 & 748 & 83 & 742 & 87 \\
\hline
\end{tabular}

rated by response. Latencies longer than 2 sec were excluded from the RT analyses. This only removed $0.3 \%$ of the correct responses across subjects.

Data from the invalid-near, invalid-object, and invalidfar conditions were initially pooled for comparison with the valid-near condition, to examine any spatial cuing effect. A two-way ANOVA of the RT data [validity (2) $\times$ response (2)] found a main effect of validity $[F(1,19)=$ $22.4, p<.001]$, with faster responses in the valid-near condition, thus indicating the effectiveness of the spatial cues. There was also a main effect of response $[F(1,19)=$ $5.2, p<.03$ ], with slower different responses, but no interaction between cuing and response $(F<1)$. A planned contrast of RTs for the valid-near versus invalid-near conditions showed that the former was significantly faster $[F(1,19)=5.8, p<.03]$. This spatial cuing effect did not interact with response $[F(1,19)=1.6$, n.s. $]$. An analysis comparing RTs in all three invalid conditions found an effect of condition $[F(2,18)=5.95, p<.01]$, which did not interact with response $(F<1)$. Comparison of RTs in the invalid-near versus invalid-far condition indicated significantly faster RTs for the near condition $[F(1,19)=9.3, p<.01]$, an effect of target separation which we will discuss later.

Crucially, comparison of RTs in the invalid-object versus invalid-far condition now found no effect of condition $(F<1)$. Any trend was for slower performance in the object condition. Thus, for the first time in our series of four experiments, no object-based advantage was observed. This was presumably due to the narrower spatial focus of attention on just the cued side.

Comparable analyses were conducted on the accuracy rates. In the analysis which examined any spatial cuing effect, by pooling the three invalid conditions for comparison with the valid-near condition, there was a main effect of validity $[F(1,19)=7.6, p<.01]$ and an interaction with response $[F(1,19)=7.9, p<.01]$, but no main effect of response $[F(1,19)=2.3$, n.s.]. Since accuracy was equivalent for same and different responses in the valid condition, the interaction with response presumably reflects the poorer accuracy for same versus different responses in just the invalid conditions.

The planned contrast of valid-near and invalid-near accuracy found an effect of spatial cuing $[F(1,19)=4.6$, $p<.05]$, which did not interact with response $(F<1)$. The analysis comparing accuracy for all three invalid conditions found an effect of response $[F(1,19)=5.7, p<.03]$, reflecting the poorer accuracy for same responses mentioned previously; there was also a main effect of condition $[F(2,18)=6.0, p<.01]$, which did not interact with response $[F(2,18)=1.25$, n.s. $]$. A planned contrast of the invalid-object and invalid-far found a significant effect $[F(1,19)=12.5, p<.002]$, reflecting a decrease in accuracy for the invalid-object trials (i.e., the opposite of the object advantage found in the previous studies). There were no other effects in the accuracy analyses.

To summarize, a spatial cuing effect was found on both RT and accuracy measures - namely, better performance 
for the near-valid condition than for all of the invalid conditions. Among the invalid conditions, RT was faster in the near than in the far and object conditions, with accuracy showing a supporting pattern. There was a cost in accuracy for the invalid-object as compared with the invalid-far condition. Since this cost was fairly small (averaging 4\%), and the supporting trend in RTs did not reach significance, we will not dwell on it. Note, however, that the present pattern of results allows a confident conclusion that there was certainly no advantage for the object condition in comparison with the far, unlike in the previous experiments. Indeed, the observed pattern was actually in the opposite direction, with performance in the three invalid conditions declining as a function of the spatial separation between target elements (near, far, and then object).

\section{Discussion}

In Experiment 4, the stimuli and task were the same as in Experiment 3, except that subjects were now precued to focus their covert attention narrowly on just one side of the display. The cue was provided by the early onset of peripheral dashes on one side, which indicated that the target elements were most likely to appear on that cued side in a near arrangement. Our purpose was to examine whether the object-based effect, previously observed across the width of the display, would be replicated when subjects had a narrow focus of covert attention on just one side of the display.

Performance was reliably fastest and most accurate for the valid-near condition, in which the target elements both appeared on the cued side. This indicates that we were successful in manipulating covert attention toward just one side of the display, and thus in inducing a spacebased form of selection. Under these circumstances, the object-based effect that had been reliably observed in the three previous experiments was no longer found. That is, when the target elements appeared far apart on opposite sides of fixation, with covert attention initially focused narrowly on just one of these sides, there was now no advantage if the target elements belonged to a common line rather than to distinct lines.

Indeed, the common-line arrangement actually showed a slight but reliable cost in accuracy, as compared with target elements appearing on opposite sides and on distinct lines. This confirms that the absence of a commonobject advantage was not due to any lack of power caused by the rarity of each invalid condition. Instead, the present absence of the common-object advantage must presumably have been due to the narrow focus of attention engendered by the cue, since that was the only change from Experiment 3.

The overall speed and accuracy in the invalid conditions declined in the following sequence: near, far, and then object conditions. One simple explanation would be that this sequence mirrors the spatial separation of the target elements, which was $1.3^{\circ}-1.6^{\circ}, 7.8^{\circ}$, and $8.1^{\circ}-8.5^{\circ}$, respectively. Such a decrease in performance with increasing separation is the usual hallmark of spatial constraints on divided visual attention (e.g., Hoffman \& Nelson, 1981; Hoffman et al., 1983). By cuing subjects to concentrate their attention narrowly on just one side of the display, we presumably induced a focused spatial form of selection. This produced the distance effects that were notably absent in the three previous experiments, where subjects presumably began with a diffuse attentional setting.

\section{GENERAL DISCUSSION}

In our introduction, we noted that previous studies have reported both space-based (Hoffman \& Nelson, 1981; Hoffman et al., 1983) and object-based (Baylis \& Driver, 1993; Duncan, 1984; Treisman et al., 1983; Vecera \& Farah, 1994) limitations on the ability to divide visual attention across two target elements. We suggested that the relation between these limitations has remained unclear, and also that many previous cases of apparently objectbased restrictions can be criticized on several methodological grounds. Our four experiments were designed to address these potential criticisms. We developed a new task to measure both space-based and object-based constraints on divided attention, in a situation where one constraint could not be reduced to the other, and where the required task did not intrinsically favor either constraint. Our first three experiments repeatedly found an object-based effect, whereby performance was better for distant elements on a common line as compared with elements a similar distance apart on distinct lines.

This effect extends previous object-based effects in several ways. First, it shows that object-based attention can operate across wide spatial extents (in the present studies, the displays subtended $13^{\circ}$ ). Previously, one might have argued that object effects are primarily found when the stimuli are too narrow to permit any effective focusing of spatial attention within them. Second, the present object-based effect was found even though subjects were given no strategic motivation to adopt an objectbased approach to selection. Unlike in previous studies, the instructions did not prespecify that one particular object should be judged (cf. Duncan, 1984; Vecera \& Farah, 1994); moreover, the event probabilities were actually weighted against object-based selection, since target elements were much more likely to appear on distinct lines than on a common line. Third, the elements to be compared were equivalent across our various conditions, thus avoiding potential stimulus confounds such as differing spatial frequencies or eccentricities (cf. Duncan, 1984; Vecera \& Farah, 1994). Finally, our last experiment provided the very first instance of an object-based effect on visual attention being eliminated when subjects were cued to adopt a narrower focus of spatial attention.

It might be suggested that our new object-based effect might actually reflect an influence of apparent depth. ${ }^{2}$ Suppose that the two lines in each display were perceived as lying on different depth planes, perhaps because of the different colors used. Target elements on a single line would then have a common perceived depth, whereas targets on different lines might require an attentional shift in 
depth, thus leading to less efficient performance (Downing \& Pinker, 1985; Gawryszewski, Riggio, Rizzolatti, \& Umiltà, 1987; Hoffman \& Mueller, 1994). Such an account in terms of perceived depth may apply to a recent study of object-based attention by Behrmann, Zemel, and Mozer (1994), who reported that shape comparisons were more efficient within one object than between two objects, using two objects arranged such that one partially occluded the other, thus providing a strong pictorial cue for a depth difference between them.

We consider any such depth account to be highly implausible for the present data, for several reasons. First, our displays were deliberately designed to eliminate any pictorial depth cues such as occlusion. Second, they appeared flat and two-dimensional to all observers by selfreport. Third, and most critically, an account in terms of apparent depth alone cannot explain the elimination of our object-based effect with narrowed spatial attention, as in Experiment 4. It was suggested during the review process (see note 2) that our use of an outer dash from each line on just one side of the display for spatial cuing might have led subjects to attend an intermediate apparent depth plane (owing to some averaging process operating on the apparent depths of the two cue dashes). Could this eliminate a depth-based object effect? There are three reasons to doubt such an account.

First, on the depth account, subjects would presumably be initially focused on the proposed intermediate plane for all four experiments, because the full displays should induce the same averaging process as would the cues. Moreover, for all experiments, it should have been advantageous to attend this intermediate plane in order to minimize any 3-D attentional shifts, since even when the targets were in the same object they were equally likely to both appear in one of the lines (on the putative near plane) or both on the other (putative far plane). Since, on this view, the intermediate plane should be attended in every experiment, it would remain unclear why the object effect was eliminated by cuing. Second, we have since replicated the elimination of the object-based effect under narrowed spatial attention, using other forms of spatial cuing (e.g., the advance presentation of an asterisk on one side, in the empty space between where the two lines subsequently appear). Hence this result is not due to any peculiarity of the dash cues.

Finally, even if subjects were led to attend an intermediate apparent depth plane only when given a lateral cue, the depth account still fails to explain the elimination of the object effect with spatial cuing. The account is predicated on the assumption that performance declines whenever attention must be shifted in apparent depth. Only one such shift from the putative intermediate plane would be required when both target elements fell on the same line, whereas two such shifts would be required when the elements fell on different lines (and thus different planes). Hence the depth account predicts that an object effect should still be found even after spatial cuing; it is therefore inconsistent with our findings.
Our use of large displays allowed us to manipulate the spatial separation between target elements substantially (it varied between $1^{\circ}$ and $8^{\circ}$ ). Nonetheless the object effect was found in the first three experiments across this wide spatial extent, with little or no evidence for any spatial constraint on performance. These results do not fit a simple space-based model of attention, on which an advantage for closer target elements is predicted. However, this does not entail that no form of spatial selectivity was involved in our task. Subjects might initially have adopted a diffuse spatial setting of attention prior to display onset in Experiments 1-3, since the target elements were known to be equally likely in the periphery on either side. When the dashed lines became segmented as distinct objects within this diffusely attended region, spatial attention might then have narrowed down more efficiently on one such object (as required in the object condition) than on an area of space that contained parts of both objects (as in the near and far conditions).

This possibility illustrates that the present objectbased results can be reconciled with the hypothesis (Tsal \& Lavie, 1988, 1993) that selection ultimately takes place within a spatial medium, albeit under the influence of object-based factors. Various previous authors (e.g., Egly et al., 1994; Gibson \& Egeth, 1994; Kramer \& Jacobson, 1991; Kramer et al., in press; Vecera, 1994) have similarly argued that visual selection may operate within a "grouped spatial array" and can thus be subject to both object-based and space-based influences. As discussed in our introduction, the only existing evidence that objectbased attention may be truly spatially invariant (i.e., Vecera \& Farah, 1994), rather than array-based, can be questioned on methodological grounds. Thus, at present the notion of selection in a grouped spatial array seems to be the more cautious and consensual view (e.g., Driver \& Baylis, 1995; Kramer et al., in press; Tsal \& Lavie, 1993), with the burden of proof still resting on any advocate of spatially invariant selection. ${ }^{3}$

Previous work has sought to determine whether selection does indeed operate on a spatial array by using essentially two methods: measuring the effects of spatially separating the stimuli (e.g., Hoffman \& Nelson, 1981; Vecera, 1994; Vecera \& Farah, 1994), or measuring any location effects on probe judgments (e.g., Tsal \& Lavie, 1993; Kim \& Cave, 1995; Kramer et al., in press; see also Weber, Kramer, \& Miller, in press, for a recent electrophysiological study of whether object-based selection has a spatial component).

As discussed above, no consistent effects of spatial separation were apparent in our own Experiments 1-3, presumably because all target events fell within the initial diffusely attended region. However, the possibility that selection ultimately operates on a grouped spatial array in our task could still be usefully examined in future research, by using a probe detection measure. Responses to subsequent probe events can be facilitated when they share the location of the previously selected item, thus demonstrating a spatial component to that preceding se- 
lection (Kim \& Cave, 1995; Kramer et al., in press; Tsal \& Lavie, 1993). The probe detection method could be adapted for the present task, to determine whether the positions occupied by the line containing the target elements become facilitated, as compared with the positions of the other line.

In the present study, we took a different approach to examining whether performance in our task could be influenced by spatially selective factors. Our use of large displays allowed us to manipulate the spatial scale of attention relative to the presented objects, which has not been possible in previous studies of object-based attention. When covert attention was cued to adopt a narrow focus on just one side, our object-based effect was eliminated, suggesting that the object-based selection may only operate within a spatially attended region. Moreover, effects of spatial separation between the target elements now became apparent, with performance falling off as the distance between target elements increased.

This change in results with narrowed attention would seem to suggest problems for some traditional accounts of object-based influences on attention. Neisser (1967), Kahneman (1973), and Driver and Baylis (1989), among others, have suggested that object-based effects are due to "preattentive" grouping processes that code the distinction between separate objects. But if the present objectbased effect were indeed due to preattentive processes of this kind, then why should it be influenced by the narrowing of spatial attention in Experiment 4, given that preattentive processes should presumably be impervious to the distribution of attention, by definition?

Fortunately, this apparent paradox arises only if one adopts a strictly sequential, stage approach to the relation between object-based processes and spatial attention. Various authors (e.g., Farah, Wallace, \& Vecera, 1993; Humphreys \& Riddoch, 1993a, 1993b; Ward, Goodrich, \& Driver, 1994) have argued instead that spatial factors and object-based factors can mutually influence each other during selection, in an interactive activation fashion (McClelland \& Rumelhart, 1981). On this view, it is possible both for object-based factors to influence the distribution of attention (as we found in Experiments 1-3), and yet also for the spatial distribution of attention to influence object-based effects (as we found in Experiment 4).

Previously, the arguments for such an interactive view have been based largely on neuropsychological findings, which show that spatial biases in attention following brain injury (as in unilateral neglect or extinction; see Robertson \& Marshall, 1993) can be modulated to some extent by object segmentation factors (Driver, 1995). In fact, if taken in isolation, such findings might actually be reconciled with the traditional serial-stage view (Kahneman, 1973; Neisser, 1967), by arguing that the neuropsychological object-based effects are due to processes that entirely precede the pathological spatial attention. However, when the neuropsychological results are taken in conjunction with the present normal findings, which show for the first time that object-based effects can be modulated by spatial attention, the interactive view seems clearly favored, because only it can accommodate all of the existing results.

To summarize, the present experiments have led to the following conclusions. Clear evidence for object-based constraints on divided visual attention can be found, while avoiding the various methodological criticisms of previous studies. Moreover, these object-based constraints can be apparent even in very wide displays. However, they are influenced by the initial spatial setting of attention. In particular, a distinction between objects that applies across wide spatial extents can become ineffective when a narrow attentional setting is initially adopted. When taken together with previous findings, these results suggest that the relation between object-based and space-based processes in selection may be interactive, with each mutually influencing the other.

\section{REFERENCES}

BAyLIS, G. C. (1994). Visual attention and objects: Two-object cost with equal convexity. Journal of Experimental Psychology: Human Perception \& Performance, 20, 208-212.

BAYLIS, G. C., \& DRIVER, J. (1992). Visual parsing and response competition: The effect of grouping factors. Perception \& Psychophysics, 51, 145-162.

BAYLIS, G. C., \& DrIVER, J. (1993). Visual attention and objects: Evidence for hierarchical coding of location. Journal of Experimental Psychology: Human Perception \& Performance, 19, 451-470.

BAYLIS, G. C., \& DRIVER, J. (1995). Obligatory edge-assignment in vision: The role of figure and part segmentation in symmetry detection. Journal of Experimental Psychology: Human Perception \& Performance, 21, 1323-1342.

Behrmann, M., Zemel, R., \& Mozer, M. C. (1994, November). Object-based attention and occlusion. Paper presented at the meeting of the Psychonomic Society, St. Louis.

COHEN, A., \& IVRY, R. (1989). Illusory conjunctions inside and outside the focus of attention. Journal of Experimental Psychology: Human Perception \& Performance, 15, 650-663.

DiXon, P., \& Just, M. A. (1978). Normalization of irrelevant dimensions in stimulus comparisons. Journal of Experimental Psychology: Human Perception \& Performance, 4, 36-46.

Downing, C. J., \& Pinker, S. (1985). The spatial structure of visual attention. In M. I. Posner \& O. S. M. Marin (Eds.), Attention and performance $X I$ (pp. 171-187). Hillsdale, NJ: Erlbaum.

DrIVER, J. (1995). Object segmentation and visual neglect. Behavioural Brain Research, 71, 135-146.

Driver, J., \& BAYlis, G. C. (1989). Movement and visual attention: The spotlight metaphor breaks down. Journal of Experimental Psychology: Human Perception \& Performance, 15, 448-456.

Driver, J., \& Baylis, G. C. (1995). One-sided edge assignment in vision: 2. Part decomposition, shape description, and attention to objects. Current Directions in Psychological Science, 4, 201-206.

DunCAN, J. (1984). Selective attention and the organization of visual information. Journal of Experimental Psychology: General, 113, 501-517.

DUNCAN, J. (1993a). Coordination of what and where in visual attention. Perception, 22, 1261-1270.

Duncan, J. (1993b). Similarity between concurrent visual discriminations: Dimensions and objects. Perception \& Psychophysics, 54, 425-430.

Egly, R., DRIVER, J., \& RAFAL, R. (1994). Shifting visual attention between objects and locations: Evidence from normal and parietal lesion subjects. Journal of Experimental Psychology: General, 123, 161-177.

Eriksen, C. W., \& Hoffman, J. E. (1972). Temporal and spatial characteristics of selective encoding from visual displays. Perception \& Psychophysics, 12, 201-204.

Eriksen, C. W., \& Hoffman, J. E. (1973). The extent of processing of 
noise elements during selective encoding from visual displays. Perception \& Psychophysics, 14, 155-160.

ERIKSEN, C. W., \& ST. JAMES, J. D. (1986). Visual attention within and around the field of focal attention: A zoom lens model. Perception \& Psychophysics, 40, 225-240.

ERIKSEN, C. W., \& YEH, Y. (1985). Allocation of attention in the visual field. Journal of Experimental Psychology: Human Perception \& Performance, 11, 583-597.

Farah, M. J., Wallace, M. A., \& Vecera, S. P. (1993). "What" and "where" in visual attention: Evidence from the neglect syndrome. In I. Robertson \& J. C. Marshall (Eds.), Unilateral neglect: Clinical and experimental studies (pp. 123-137). Hillsdale, NJ: Erlbaum.

GaWryszewski, L., Riggio, L., Rizzolatti, G., \& Umiltà, C. (1987). Movements of attention in the three spatial dimensions and the meaning of "neutral" cues. Neuropsychologia, 25, 19-29.

GiBson, B. S. (1994). Visual attention and objects: One versus two or convex versus concave? Journal of Experimental Psychology: Human Perception \& Performance, 20, 203-207.

GiBson, B. S., \& EGETH, H. (1994). Inhibition of return to object-based and environment-based location. Perception \& Psychophysics, 55, 323-339.

Hoffman, J. E., \& Mueller, S. (1994, November). An in depth look at visual attention. Paper presented at the meeting of the Psychonomic Society, St. Louis.

Hoffman, J. E., \& Nelson, B. (1981). Spatial selectivity in visual search. Perception \& Psychophysics, 30, 283-290.

Hoffman, J. E., Nelson, B., \& Houck, M. R. (1983). The role of attentional resources in automatic detection. Cognitive Psychology, 15, 379-410.

HumphREys, G. W., \& RidDOCH, M. J. (1993a). Interactions between object and space systems revealed through neuropsychology. In D. E. Meyer \& S. Kornblum (Eds.), Attention and performance XIV (pp. 143-162). Cambridge, MA: MIT Press.

HUMPHREYS, G. W., \& RiDDOCH, M. J. (1993b). Interactive attentional systems and unilateral visual neglect. In I. H. Robertson \& J. C. Marshall (Eds.), Unilateral neglect: Clinical and experimental studies (pp. 139-167). Hove, U.K.: Erlbaum.

Kahneman, D. (1973). Attention and effort. Englewood Cliffs, NJ: Prentice-Hall.

Kanwisher, N., \& Driver, J. (1992). Objects, attributes, and visual attention: Which, what, and where. Current Directions in Psychological Science, 1, 26-31.

Kim, M. S., \& CAve, K. (1995). Spatial attention in visual search for features and feature conjunctions. Psychological Science, 6, 376-380.

Kramer, A. F., \& Jacobson, A. (1991). Perceptual organization and focused attention: The role of objects and proximity in visual processing. Perception \& Psychophysics, 50, 267-284.

Kramer, A. F., Weber, T. A., \& Watson, S. E. (in press). Object-based attentional selection: Grouped arrays or spatially-invariant representations? Journal of Experimental Psychology: General.

LaBerge, D., Brown, V., Carter, M., Bash, D., \& Hartley, A. (1991). Reducing the effects of adjacent distractors by narrowing attention. Journal of Experimental Psychology: Human Perception \& Performance, 17, 65-76.

Mack, A., Tang, B., Tuma, R., Kahn, S., \& Rock, I. (1992). Perceptual organization and attention. Cognitive Psychology, 24, 475-50l.

MarR, D., \& Nishimara, H. K. (1978). Representation and recognition of the spatial organization of three-dimensional shapes. Proceedings of the Royal Society of London: Series B, 200, 269-294.

McClelland, J. L., \& Rumelhart, D. E. (1981). An interactive model of context effects in letter perception: $I$. An account of basic findings. Psychological Review, 88, 375-407.

Miller, J., \& BAUER, D. W. (1981). Irrelevant differences in the "same"-"different" task. Journal of Experimental Psychology: Human Perception \& Performance, 7, 196-207.

NeISSER, U. (1967). Cognitive psychology. New York: Appleton.

PosNer, M. I. (1980). Orienting of attention. Quarterly Journal of Experimental Psychology, 32, 3-25.

Robertson, I. H., \& MARShall, J. C. (EDS.) (1993). Unilateral neglect: Clinical and experimental studies. Hove, U.K.: Erlbaum.

RUBIN, E. (1921). Visuell wahrgenommene figuren [Visual figure and ground]. Copenhagen: Glydendal.

SCHNEIDER, W. (1988). Micro Experimental Laboratory: An integrated system for IBM PC compatibles. Behavior Research Methods, Instruments, \& Computers, 20, 206-217.

Treisman, A., \& Gelade, G. (1980). A feature-integration theory of attention. Cognitive Psychology, 12, 97-136.

Treisman, A., Kahneman, D., \& Burkell, J. (1983). Perceptual objects and the cost of filtering. Perception \& Psychophysics, 33, 527532.

Tsal, Y., \& LAvIE, N. (1988). Attending to color and shape: The special role of location in selective visual processing. Perception \& Psychophysics, 44, 15-21.

TSAL, Y., \& LAVIE, N. (1993). Location dominance in attending to color and shape. Journal of Experimental Psychology: Human Perception \& Performance, 19, 131-139.

VECERA, S. (1994). Grouped locations and object-based attention: Comment on Egly, Driver, and Rafal (1994). Journal of Experimental Psychology: General, 123, 316-320.

VECERA, S., \& FARAH, M. (1994). Does visual attention select objects or locations? Journal of Experimental Psychology: General, 123, 146-160

WARD, R., GoOdrich, S., \& Driver, J. (1994). Grouping reduces visual extinction: Neuropsychological evidence for weight-linkage in visual selection. Visual Cognition, 1, 101-129.

WATT, R. J. (1988), Visual processing: Computational, psychophysical, and cognitive research. Hillsdale, $\mathrm{NJ}$ : Erlbaum.

Weber, T. A., Kramer, A. F., \& Miller, G. (in press). Selective processing of superimposed and separated objects: An electrophysiological analysis of object-based attentional selection. Biological Psychology.

\section{NOTES}

1. A few studies of object-based attention using measures other than dual tasks (e.g., studies which examine the effects of grouping on distractor interference; Baylis \& Driver, 1992; Kramer \& Jacobson, 1991) have manipulated spatial separation as well as segmentation. However, they have not done so over a large range (spatial separation between target and distractor typically varied within $1^{\circ}$ of visual angle) and have usually confounded spatial separation with distractor eccentricity. In any case, our review is concerned specifically with limits on divided attention.

2. Our thanks to Art Kramer for raising this issue.

3. The spatially invariant form of object-based selection was suggested as one of multiple possible forms for selection (i.e., spatially invariant selection was suggested in addition to grouped array and purely spatial selection; Vecera \& Farah, 1994). Thus, spatially invariant and array-based forms of selection need not be mutually exclusive. However, for reasons of parsimony, an account of existing evidence in terms of just one form of selection is preferable to multiple forms until new evidence proves otherwise.

(Manuscript received March 20, 1995; revision accepted for publication February 17, 1996.) 